\title{
Latanoprostene Bunod Ophthalmic Solution 0.024\%: A Review in Open-Angle Glaucoma and Ocular Hypertension
}

\author{
Sheridan M. Hoy ${ }^{1}$
}

Published online: 14 May 2018

(C) Springer Nature 2018, corrected publication May/2018

\begin{abstract}
Latanoprostene bunod ophthalmic solution $0.024 \%$ (hereafter referred to as latanoprostene bunod $0.024 \%$ ) $\left[\mathrm{V} y z u l t a^{\mathrm{TM}}\right]$ is a nitric oxide (NO)-donating prostaglandin $\mathrm{F}_{2 \alpha}$ analogue approved in the USA for the reduction of intraocular pressure (IOP) in patients with open-angle glaucoma $(\mathrm{OAG})$ or ocular hypertension. It is thought to lower IOP by increasing aqueous humour outflow through the uveoscleral pathway (mediated by latanoprost acid) and increasing the facility of aqueous humour outflow through the trabecular meshwork pathway (mediated by NO). Results from two multinational, phase III studies (APOLLO and LUNAR) and a pooled analysis of these studies demonstrated the noninferiority of latanoprostene bunod $0.024 \%$ to timolol ophthalmic solution $0.5 \%$ (hereafter referred to as timolol $0.5 \%$ ) in terms of IOP-lowering efficacy over 3 months in patients with OAG or ocular hypertension, with the superiority of latanoprostene bunod $0.024 \%$ over timolol $0.5 \%$ subsequently demonstrated in APOLLO and the pooled analysis. Moreover, there was no apparent loss of IOP-lowering effect in subsequent safety extension periods of up to 9 months. The
\end{abstract}

The original version of this article was revised due to a retrospective Open Access request.

The manuscript was reviewed by: $\boldsymbol{A}$. L. Robin, Department of Ophthalmology, University of Michigan, Ann Arbor, MI and Departments of Ophthalmology and International Health, Johns Hopkins University, Baltimore, MD, USA; C. B. Toris, Case Western Reserve University, Cleveland, OH, USA; T. Yamamoto, Department of Ophthalmology, Gifu University Graduate School of Medicine, Gifu, Japan.

Sheridan M. Hoy

demail@springer.com

1 Springer, Private Bag 65901, Mairangi Bay, Auckland 0754, New Zealand
IOP-lowering efficacy seen in APOLLO and LUNAR was confirmed in a phase III study (JUPITER) in Japanese patients, with IOP reductions observed early (week 4) and maintained over the longer-term (12 months). Latanoprostene bunod $0.024 \%$ was well tolerated over up to 12 months in these studies, with most ocular treatmentemergent adverse events (TEAEs) being mild to moderate in severity. Thus, current evidence indicates once-daily latanoprostene bunod $0.024 \%$ is an effective and well tolerated treatment option for the reduction of IOP in adults with OAG or ocular hypertension.

\section{Latanoprostene Bunod Ophthalmic Solution} $0.024 \%$ : clinical considerations

NO-donating prostaglandin $\mathrm{F}_{2 \alpha}$ analogue; instilled once daily in the evening

Thought to lower IOP via a dual mechanism of action

Significantly more effective than timolol $0.5 \%$ in lowering IOP in adults

IOP-lowering benefits were maintained over the longer-term (12 months)

Well tolerated, with most ocular TEAEs being mild or moderate in severity

\section{Introduction}

Maintaining intraocular pressure (IOP) entails balancing aqueous humour production (in the ciliary body) and outflow (via the trabecular meshwork and uveoscleral pathways) [1]. Resistance to aqueous humour outflow through the trabecular meshwork is increased in patients with 
primary open-angle glaucoma (OAG), resulting in elevated IOP, and lowering IOP (by enhancing outflow or decreasing production) is currently the only proven therapeutic approach to preserving visual function in this patient population [1-3].

A single topical ophthalmic agent, most commonly a prostaglandin analogue (PGA), is typically used for the initial treatment of primary OAG $[1,4]$. However, many patients require treatment with more than one agent (preferably agents with differing, but complementary, mechanisms of action) to adequately control IOP and thus prevent disease progression [1, 3, 4]. Several recently developed agents lower IOP by targeting aqueous humour outflow via the trabecular meshwork pathway $[1,5]$. One such agent is the once-daily, nitric oxide (NO)-donating prostaglandin $\mathrm{F}_{2 \alpha}$ analogue latanoprostene bunod ophthalmic solution $0.024 \%$ (hereafter referred to as latanoprostene bunod $0.024 \%$ ) $\left[\mathrm{Vyzulta}^{\mathrm{TM}}\right]$. Latanoprostene bunod is a single molecule with two active metabolites, each with its own mechanism of action [1, 5] (Sect. 2.1). This article discusses pharmacological, therapeutic efficacy and tolerability data relevant to the use of latanoprostene bunod $0.024 \%$ for the reduction of IOP in patients with $\mathrm{OAG}$ or ocular hypertension.

\section{Pharmacological Properties}

\subsection{Mechanism of Action}

Following topical administration, latanoprostene bunod is hydrolysed (by corneal esterases) to the prostanoid FP receptor agonist latanoprost acid (active metabolite) and butanediol mononitrate, which is further metabolized to 1,4-butanediol and NO (active metabolite) [1] (Fig. 1).

Latanoprost acid increases matrix metalloproteinase (MMP)-1, MMP-3 and MMP-9 expression in the ciliary muscle, promoting the remodelling of its extracellular matrix and, subsequently, increased aqueous humour outflow through the uveoscleral pathway [1]. MMPs may also play a minor role in augmenting aqueous humour outflow facility through the trabecular meshwork pathway by remodelling the extracellular matrix of the trabecular meshwork $[1,6]$.

$\mathrm{NO}$ is an endogenous signalling molecule known for its role as a mediator of smooth muscle relaxation and vasodilation [1]. NO synthases are present in various ocular tissues (e.g. trabecular meshwork, Schlemm's canal, ciliary body) of healthy volunteers, but reduced in these tissues in patients with primary OAG, suggesting reduced NO production may contribute to elevated IOP [6]. Activation of the soluble guanylate cyclase (sGC)/cyclic guanosine monophosphate (cGMP)/protein kinase $\mathrm{G}$ pathway by $\mathrm{NO}$

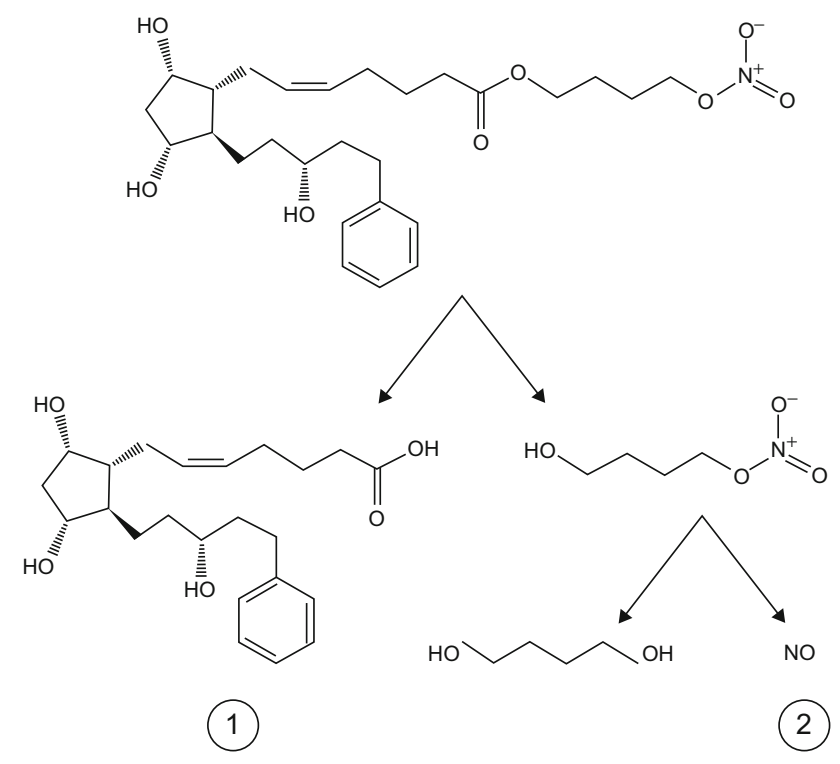

Fig. 1 Chemical structure of latanoprostene bunod and its metabolism to latanoprost acid (1) and butanediol mononitrate, with the subsequent release of nitric oxide (2) and 1,4-butanediol (an inactive metabolite). Reproduced from Kawase et al. [14] with permission

results in several downstream effects, including reduced intracellular calcium levels and Rho-associated protein kinase inhibition, which lead to myosin light chain (MLC)2 dephosphorylation [1, 6, 7]. This, in turn, promotes actin cytoskeleton rearrangement, thereby decreasing cell contractility and volume, and, thus, improving the facility of aqueous humour outflow through the trabecular meshwork pathway $[1,6,7]$.

\subsection{Pharmacodynamic Profile}

The pharmacodynamic properties of latanoprostene bunod have been characterised in vitro and in animal models of IOP. In human trabecular meshwork cells (HTMCs), latanoprostene bunod (half maximal effective concentration of $1.5 \mu \mathrm{mol} / \mathrm{L}$ ) significantly ( $p<0.05$ vs. control) increased cGMP levels in a dose- and sGC-dependent manner [8]. Moreover, latanoprostene bunod reduced endothelin-1-induced MLC-2 phosphorylation ( $p<0.05$ vs. endothelin-1) and actin stress fibres (an indicator of cytoskeletal contractility), as well as the localization of vinculin at focal adhesions (an indicator of cell attachment). Equimolar concentrations of latanoprost had a minimal effect on these parameters [8].

Inducing cell contractility (as seen with endothelin-1 and thrombin) increases HTMC resistance, while decreasing cell contractility decreases HTMC resistance [8]. Latanoprost demonstrated synergy with a NO donor in reducing endothelin-1-induced HTMC resistance; both latanoprost plus the NO donor and latanoprostene bunod alone were associated with a significantly $(p<0.05)$ 
greater reduction in HTMC resistance than latanoprost alone [8].

Latanoprostene bunod, but not latanoprost, demonstrated an IOP-lowering effect in F-prostanoid receptor knockout mice (a model that is insensitive to PGAs [7]) [data from an abstract] [9], suggesting that in this model the effect is due to the action of NO on the trabecular meshwork pathway [7]. Latanoprostene bunod also demonstrated IOP-lowering activity in various ocular hypertension or glaucoma animal models and lowered IOP to a greater extent than an equimolar concentration of latanoprost [10].

\subsection{Pharmacokinetic Profile}

Following ocular instillation, latanoprostene bunod is rapidly hydrolysed [half-life $\left(\mathrm{t}_{1 / 2}\right)$ in rabbit and primate corneal homogenate of 0.05 and 0.40 min compared with 0.28 and $5.2 \mathrm{~min}$ for latanoprost] [7].

No ocular distribution studies have been conducted in humans [11]. In a study in monkeys, latanoprostene bunod was not detected at any timepoint (consistent with its rapid hydrolysis) [10]. Following a single topical dose of latanoprostene bunod $0.012 \%$, maximum concentrations $\left(\mathrm{C}_{\max }\right)$ of latanoprost acid in the cornea, aqueous humour and iris-ciliary body of rabbits and monkeys were reached within $0.5-1 \mathrm{~h}$ (which was similar to that seen with an equimolar concentration of latanoprost), suggesting rapid distribution. The $t_{1 / 2}$ of latanoprost acid was $1.8-4.6 \mathrm{~h}$ for latanoprostene bunod and 1.1-3.0 h for latanoprost across the two species [10]. As NO has a short $t_{1 / 2}(\approx 2 \mathrm{~s}$ in extravascular tissue $)$ and is highly diffusible, it is difficult to measure in vivo [7]; however, levels of NO's downstream effector cGMP in the aqueous humour and iris-ciliary body of rabbits were significantly $(p<0.05)$ increased from baseline following the ocular instillation of latanoprostene bunod, but not latanoprost [10].

In 22 healthy volunteers who received latanoprostene bunod $0.024 \%$ once daily (in the morning) for 28 days, there were no quantifiable plasma concentrations of latanoprostene bunod [lower limit of quantitation (LLOQ) of $10.0 \mathrm{pg} / \mathrm{mL}$ ) or butanediol mononitrate (LLOQ of $200 \mathrm{pg} / \mathrm{mL}$ ) post instillation on days 1 and 28 [11]. Post dose, mean plasma $\mathrm{C}_{\max }$ values of latanoprost acid (LLOQ of $30 \mathrm{pg} / \mathrm{mL}$ ) were 59.1 and $51.1 \mathrm{pg} / \mathrm{mL}$ on the respective days and the mean times to $C_{\max }$ were approximately $5 \mathrm{~min}$ on both days. Upon reaching the systemic circulation, latanoprost acid is predominately metabolized via fatty acid $\beta$-oxidation in the liver to the 1,2-dinor and 1,2,3,4-tetranor metabolites. Latanoprost acid is rapidly eliminated from human plasma, with the plasma concentration dropping below the LLOQ in most patients by $15 \mathrm{~min}$ post instillation of latanoprostene bunod $0.024 \%$ [11].

\section{Therapeutic Efficacy}

The IOP-lowering efficacy of latanoprostene bunod $0.024 \%$ in adults with OAG or ocular hypertension has been evaluated in several studies, with this section focusing on two randomized, double-masked, active comparatorcontrolled [timolol ophthalmic solution $0.5 \%$ (hereafter referred to as timolol $0.5 \%)]$, multinational, phase III studies (APOLLO [12] and LUNAR [13]) [Sect. 3.1]. Efficacy data from a noncomparative, open-label, multicentre, phase III study (JUPITER) [14], which was primarily designed to evaluate safety, are also reviewed (Sect. 3.2). Overall, there were no clinical differences between elderly patients and other adult patients in terms of efficacy, according to the US prescribing information [11].

A latanoprostene bunod concentration of $0.024 \%$ (instilled once daily in the evening) was identified as the lower of the two most effective concentrations assessed in a 4-week, randomized, single-masked, phase II, doseranging study (VOYAGER) [15] in adults with OAG or ocular hypertension. Its use resulted in a statistically significant $(p=0.005)$ reduction from baseline in least-squares mean (LSM) diurnal IOP at day 28 (primary endpoint) compared with latanoprost ophthalmic solution $0.005 \%$ (between-group difference of $1.23 \mathrm{mmHg}$ ) [15]. The 24-h efficacy of latanoprostene bunod $0.024 \%$ (instilled once daily in the evening) compared with timolol $0.5 \%$ (instilled twice daily) was assessed in a 4-week, randomized, openlabel, crossover, phase II study (CONSTELLATION) in 21 adults with ocular hypertension or early primary OAG [16]. IOP was measured at baseline and the end of each 4-week period in a 24-h sleep laboratory. Latanoprostene bunod $0.024 \%$ was associated with a significant $(p \leq 0.002)$ reduction from baseline in diurnal and nocturnal IOP, whereas timolol $0.5 \%$ was associated with a significant $(p<0.001)$ reduction from baseline in diurnal IOP only. The reduction in nocturnal IOP was significantly ( $p=$ 0.004 ) higher with latanoprostene bunod $0.024 \%$ than timolol $0.05 \%$. Moreover, latanoprostene bunod therapy resulted in a significantly greater diurnal ocular perfusion pressure compared with baseline $(p \leq 0.006)$ and nocturnal ocular perfusion pressure compared with timolol $0.5 \%$ ( $p=$ $0.01)$ [16].

\subsection{APOLLO and LUNAR}

APOLLO [12] and LUNAR [13] consisted of a 3-month, active-controlled period followed by a 3-month (LUNAR) or 9-month (APOLLO) open-label safety extension period 
and enrolled patients aged $\geq 18$ years with a diagnosis of OAG (including pigmentary or pseudoexfoliative OAG) or ocular hypertension in one or both eyes. IOP was assessed at screening and at $8 \mathrm{AM}, 12 \mathrm{PM}$ and $4 \mathrm{PM}$ at baseline, with patients required to have an IOP of $\geq 26 \mathrm{mmHg}$ at $\geq 1$ timepoint, $\geq 24 \mathrm{mmHg}$ at $\geq 1$ timepoint and $\geq 22 \mathrm{mmHg}$ at 1 timepoint in the same eye; an IOP of $\leq 36 \mathrm{mmHg}$ at all three baseline timepoints in both eyes; and a best-corrected visual acuity (BCVA) of +0.7 logarithm of the minimum angle of resolution (Snellen equivalent of $\approx 20 / 100$ ) or better in either eye. The eye with the highest IOP was designated the study eye. Patients received latanoprostene bunod $0.024 \%$ (instilled once daily in the evening) or timolol $0.5 \%$ (instilled twice daily) for 12 weeks; those who received IOP-lowering therapy at or within 30 days of screening $(72 \%)$ were required to undergo a $\leq 28$-day washout period prior to randomization $[12,13]$. During the open-label safety extension period, all patients received latanoprostene bunod $0.024 \%$ [17]. Patients had a mean duration of exposure to latanoprostene bunod $0.024 \%$ of 90.3 days during the active-controlled period and 231.9 days during the active-controlled and open-label safety extension periods, and a mean duration of exposure to timolol $0.5 \%$ of 90.4 days [17].

Baseline patient demographics and eye characteristics were comparable between the latanoprostene bunod $0.024 \%$ and timolol $0.5 \%$ groups in both APOLLO [12] and LUNAR [13]. Mean baseline diurnal IOP in the latanoprostene bunod $0.024 \%$ and timolol $0.5 \%$ groups was 26.7 and $26.5 \mathrm{mmHg}$ in APOLLO [12] and 26.6 and $26.4 \mathrm{mmHg}$ in LUNAR [13]. The primary endpoint was the study eye IOP at three timepoints ( 8 AM, 12 PM and 4 PM) at weeks 2, 6 and $12[12,13]$. The primary objective was to assess the noninferiority of latanoprostene bunod $0.024 \%$ versus timolol $0.5 \%$ in terms of IOP reduction at each timepoint throughout the 12 weeks of therapy. If noninferiority was established, the secondary objective was to assess the superiority of latanoprostene bunod $0.024 \%$ versus timolol $0.5 \%$. Key secondary endpoints were the proportion of patients achieving an IOP reduction of $\geq 25 \%$ and the proportion of patients achieving an IOP of $\leq 18 \mathrm{mmHg}$ at all nine timepoints. Analyses were conducted in the intent-to-treat population [12, 13].

The IOP-lowering efficacy of latanoprostene bunod $0.024 \%$ was noninferior to that of timolol $0.5 \%$ in APOLLO [12], LUNAR [13] and a pooled analysis [17] of these studies, as the upper limit of the $95 \%$ CIs for the between-group differences in the primary endpoint did not exceed the predefined limits of $1.5 \mathrm{mmHg}$ at all 9 timepoints and $1.0 \mathrm{mmHg}$ for $\geq 5$ of the 9 timepoints. Moreover, in APOLLO [12] and the pooled analysis [17], latanoprostene bunod $0.024 \%$ was superior to timolol $0.5 \%$, as the upper limit of the $95 \%$ CIs for the between- group difference in the primary endpoint did not exceed the predefined limit of $0 \mathrm{mmHg}$ at all nine timepoints. In LUNAR, the superiority criteria were met for all of the timepoints except the $8 \mathrm{AM}$ timepoint at week 2 [13]. Sensitivity analyses demonstrated that the results of the primary analyses were robust [12, 13, 17], with the findings unaffected by prior treatment status $[12,13]$, patient age ( $<65$ vs. $\geq 65$ years) [12] or the concurrent use of $\beta$-blockers $[12,13]$.

LSM of the mean IOP was significantly $(p<0.05)$ lower with latanoprostene bunod $0.024 \%$ than timolol $0.5 \%$ at all timepoints in APOLLO [12], LUNAR [13] and the pooled analysis [17], apart from at the 8 AM timepoint at week 2 in LUNAR (Table 1). Moreover, at all nine timepoints, significantly greater proportions of latanoprostene bunod $0.024 \%$ than timolol $0.5 \%$ recipients achieved an IOP reduction of $\geq 25 \%$ in APOLLO (34.9 vs. $19.5 \%$; $p=$ 0.001 ) [12], LUNAR (31.0 vs. $18.5 \% ; p=0.007$ ) [13] and the pooled analysis (32.9 vs. $19.0 \% ; p<0.001)$ [17], and an IOP of $\leq 18 \mathrm{mmHg}$ in APOLLO (22.9 vs. $11.3 \% ; p=$ 0.005 ) [12] and the pooled analysis (20.2 vs. $11.2 \%$; $p=$ $0.001)$ [17], but not LUNAR (17.7 vs. 11.1\%) [13]. In a post hoc analysis of pooled data [17], significantly $(p<$ 0.001 ) more latanoprostene bunod $0.024 \%$ than timolol $0.5 \%$ recipients achieved a week 12 IOP of $\leq 18, \leq 17$, $\leq 16$, $\leq 15$ and $\leq 14 \mathrm{mmHg}$.

Latanoprostene bunod $0.024 \%$ was associated with a significantly $(p \leq 0.025)$ greater mean reduction from baseline in mean IOP across the nine timepoints than timolol $0.5 \%$ (7.7-9.1 vs. $6.6-8.0 \mathrm{mmHg}$ in APOLLO; 7.5-8.8 vs. $6.6-7.9 \mathrm{mmHg}$ in LUNAR), except for the 8 AM timepoint at week 2 in LUNAR $[12,13]$.

Mean diurnal IOP was significantly lower with latanoprostene bunod $0.024 \%$ than timolol $0.5 \%$ at each visit in APOLLO (week 2: 18.2 vs. $19.5 \mathrm{mmHg}$; week 6: 18.1 vs. $19.3 \mathrm{mmHg}$; week $12: 18.2$ vs. $19.4 \mathrm{mmHg}$; all $p<0.001$ ) [12] and LUNAR (week 2: 18.6 vs. $19.2 \mathrm{mmHg}$; week 6: 18.2 vs. $19.1 \mathrm{mmHg}$; week 12: 18.1 vs. $19.3 \mathrm{mmHg}$; all $p \leq 0.034$ ) [13]. Of note, in a post hoc analysis of pooled data, the LSM percentage reduction from baseline to week 12 in mean diurnal IOP was significantly greater with latanoprostene bunod $0.024 \%$ than timolol $0.5 \%$ (32.0 vs. $27.6 \% ; p<0.001)$ [17].

In the pooled analysis, the beneficial effects of latanoprostene bunod $0.024 \%$ therapy were maintained during the open-label safety extension periods of APOLLO and LUNAR, with no apparent loss of IOP-lowering effect over time [17]. Mean reductions from baseline to months 6, 9 and 12 in mean diurnal IOP were $8.6,8.5$ and $8.8 \mathrm{mmHg}$, respectively, in patients who received latanoprostene bunod $0.024 \%$ during the active-controlled period and 8.5 , 8.7 and $8.7 \mathrm{mmHg}$, respectively, in those who received timolol $0.5 \%$ during the active-controlled period (all $p<$ 
0.001). The patients who received timolol $0.5 \%$ during the active-controlled period showed an additional 1.1-1.2 mmHg reduction in mean diurnal IOP at months 6 , 9 and 12 that was significant $(p \leq 0.009)$ versus week 12 . During the open-label safety extension periods, the percentage reductions from baseline (32-34\% [18]) were similar to the percentage reductions reported for therapy with latanoprostene bunod $0.024 \%$ at week 12 [17].

\subsection{JUPITER}

JUPITER [14] enrolled Japanese patients aged $\geq 20$ years with a diagnosis of OAG (including normotensive, pigmentary or pseudoexfoliative OAG) or ocular hypertension in one or both eyes. Patients were required to have a mean/median IOP of $\geq 15$ and $\leq 36 \mathrm{mmHg}$ at $10 \mathrm{AM}$ in one or both eyes, and an IOP of $\leq 36 \mathrm{mmHg}$ in both eyes at baseline; and a corrected visual acuity or BCVA of $\geq 0.5$ in both eyes. The eye with the highest IOP was designated the study eye. Patients received latanoprostene bunod $0.024 \%$ (instilled once daily in the evening) for 12 months; those receiving IOP-lowering therapy at screening $(90 \%)$ were required to undergo a 5-28-day washout period prior to baseline. The mean and median duration of exposure to latanoprostene bunod $0.024 \%$ was 351.5 and 364.0 days. Mean baseline IOP in the study and fellow treated eyes was 19.6 and $18.7 \mathrm{mmHg}$. Analyses were conducted in the safety population [14].

The IOP-lowering benefits obtained with latanoprostene bunod $0.024 \%$ in APOLLO [12] and LUNAR [13] were confirmed in JUPITER [14], with reductions in IOP seen early and maintained over the longer-term (12 months) in Japanese patients. Significant $(p<0.001)$ reductions from baseline in mean IOP of $22.0 \%(4.3 \mathrm{mmHg})$ in the study eyes $(n=130)$ and $19.5 \%(\approx 3.6 \mathrm{mmHg})$ in the fellow treated eyes $(n=126)$ were seen as early as week 4 , with significant $(p<0.001)$ reductions from baseline in this endpoint of $>22 \%$ and $>20 \%$ in the respective eyes seen at every subsequent visit (i.e. every 4 weeks from week 4 to week 52). At week 52, mean IOP in the study eyes and the fellow treated eyes was 14.4 and $14.4 \mathrm{mmHg}$; the reductions from baseline in mean IOP were $26.3 \%$ $(5.3 \mathrm{mmHg})$ and $23.0 \%(\approx 4.3 \mathrm{mmHg})$. The proportions of patients achieving a reduction from baseline in IOP of $\geq$ $5 \mathrm{mmHg}$ in the study eye were $42.3 \%$ at week $4,48.1 \%$ at week 8 and 52.3-64.2\% from weeks 12-52 [14].

\section{Tolerability}

Latanoprostene bunod $0.024 \%$ was well tolerated over up to 12 months in adults with OAG or ocular hypertension participating in APOLLO [12] and LUNAR [13]. In the 
pooled analysis [17] of these studies, the overall incidence of ocular adverse events was comparable with those of other PGAs and no new ocular adverse events were reported. Overall, there were no clinical differences between elderly patients and other adult patients in terms of safety [11].

In the pooled analysis [17], $21.6 \%$ of 811 latanoprostene bunod $0.024 \%$ recipients (i.e. who received latanoprostene bunod $0.024 \%$ during the active-controlled period and/or the open-label safety extension period) and $12.5 \%$ of 271 timolol $0.5 \%$ recipients (i.e. who received timolol $0.5 \%$ during the active-controlled period) experienced $\geq 1$ ocular treatment-emergent adverse event (TEAE) in their study eye. Most of these TEAEs were considered at least possibly related to the study medication $(80.2$ and $86.5 \%$ in the latanoprostene bunod $0.024 \%$ and timolol $0.5 \%$ groups) and mild or moderate in severity (97.0 and 97.3\%). Similar results were reported for the fellow treated eyes [17].

TEAEs typical of PGA therapy include conjunctival hyperaemia, eyelash growth and iris hyperpigmentation $[14,17]$. The most frequently reported (occurring in $\geq 3 \%$ of patients in either the latanoprostene bunod $0.024 \%$ or timolol $0.5 \%$ group) ocular TEAEs in the study eyes of the respective groups were conjunctival hyperaemia (5.9 and $1.1 \%$ of patients), eye irritation (4.6 and 2.6\%) and eye pain (3.6 and $2.2 \%$ ) [17]. The nature and incidence of the most frequently reported ocular TEAEs in the fellow treated eyes were generally similar to those in the study eyes. At baseline (i.e. prior to initiation of the study medication), the incidence of conjunctival hyperaemia (which was mostly mild to moderate in severity) as assessed by the investigator in the study eyes of the latanoprostene bunod $0.024 \%$ and timolol $0.5 \%$ groups was high (32.6 and 34.3\%). The incidence in the latanoprostene bunod $0.024 \%$ group increased from baseline at week 2 (49.0\% of patients) and was generally stable across the remainder of the study (44.2-48.7\%), while the incidence in the timolol $0.5 \%$ group was generally stable (35.8-38.9\%), but increased when patients entered the open-label safety extension period and received latanoprostene bunod $0.024 \%$ (45.2-50.0\%). In terms of adverse events of special interest (eyelid and iris pigmentation, eyelash growth), eyelash growth considered probably related to the study medication was reported in both eyes of one latanoprostene bunod $0.024 \%$ recipient and iris hyperpigmentation considered definitely related to the study medication was reported in both eyes of another latanoprostene bunod $0.024 \%$ recipient. No adverse events of special interest were reported in patients receiving timolol $0.5 \%$ [17].

Severe ocular TEAEs were reported in the study eyes of six latanoprostene bunod $0.024 \%$ recipients (allergic conjunctivitis, blepharospasm, conjunctival hyperaemia, eyelid tumour, increased IOP, retinal vein occlusion) and one timolol $0.5 \%$ recipient (instillation-site pain) and in the fellow treated eyes of five latanoprostene bunod $0.024 \%$ recipients (allergic conjunctivitis, conjunctival hyperaemia, foreign body in the eye, increased IOP, scleritis) and two timolol $0.5 \%$ recipients (increased IOP, instillation-site pain) [17]. There were no serious TEAEs in the study eye in either treatment group; one patient in the latanoprostene bunod $0.024 \%$ group experienced a serious ocular TEAE (dislocation of the intraocular lens) in the fellow treated eye. At least one non-ocular serious TEAE (not considered to be related to the study medication) was reported in $2.0 \%$ of latanoprostene bunod $0.024 \%$ recipients and $0.7 \%$ of timolol $0.5 \%$ recipients [17].

Few patients discontinued treatment because of a TEAE (2.1 and $4.5 \%$ of patients receiving latanoprostene bunod $0.024 \%$ or timolol $0.5 \%$ ), according to the pooled analysis [17]. Ocular TEAEs led to treatment discontinuation in $1.4 \%$ of latanoprostene bunod $0.024 \%$ recipients and $1.5 \%$ of timolol $0.5 \%$ recipients, with 0.6 and $0.4 \%$ of patients discontinuing treatment because of non-ocular TEAEs. Two patients in the latanoprostene bunod $0.024 \%$ group died (with one death occurring after study exit); neither death (cardiac arrest and sepsis) was considered to be related to the study medication [17].

Non-ocular TEAEs occurred in $<2 \%$ of patients in either treatment group, with the most frequent being headache (occurring in five patients in the latanoprostene bunod $0.024 \%$ group and five patients in the timolol $0.5 \%$ group) [17]. None of the non-ocular TEAEs were considered definitely related to the study medication, apart from one event of dysgeusia in one patient receiving latanoprostene bunod $0.024 \%$ [17].

BCVA and vital signs were comparable between the latanoprostene bunod $0.024 \%$ and timolol $0.5 \%$ groups during the active-controlled period and there were no safety concerns in terms of BCVA, ocular signs or vital signs during the openlabel safety extension period [17]. No clinically significant systemic TEAEs were reported [17].

Data from JUPITER showed that latanoprostene bunod $0.024 \%$ was well tolerated over 12 months in Japanese adults with OAG or ocular hypertension [14]. At least one ocular TEAE occurred in 58.5\% of 130 study eyes and $61.9 \%$ of 126 fellow treated eyes, with most considered at least possibly related to the study medication and all being mild to moderate in severity; no severe ocular TEAEs were reported. The most frequently reported (occurring in $\geq 4.0 \%$ of study or fellow treated eyes) TEAEs were conjunctival hyperaemia (occurring in 17.7 and $16.7 \%$ of eyes), eyelash growth (16.2 and 16.7\%), eye irritation (11.5 and $11.9 \%$ ), eye pain (10.0 and $10.3 \%)$ and iris hyperpigmentation (3.8 and $4.0 \%$ ). Of note, the proportion of study and fellow treated eyes with investigator-assessed conjunctival hyperaemia remained low, with only a $2.8 \%$ increase in both the study and fellow treated eye groups at week 52 compared with baseline (i.e. prior to initiation of the study 
medication) values (15.4 and 14.3\%). Most cases were mild in severity. In terms of adverse events of special interest, a clear or possible iris pigmentation increase from baseline at week 52 was reported in 10.0 and $14.6 \%$ of 130 study eyes and in 8.8 and $13.6 \%$ of 125 fellow treated eyes based on the analysis of iris photographs. Four patients discontinued JUPITER because of TEAEs, none of which were considered related to the study medication. At least one non-ocular TEAE occurred in 51.5\% of 130 patients, with nasopharyngitis (32.3\%), influenza (3.8\%), eczema (3.1\%) and osteoporosis $(2.3 \%)$ being the most frequently reported. None were considered related to latanoprostene bunod $0.024 \%$. There were no safety concerns in terms of vital or ocular signs in JUPITER, and corrected visual acuity appeared to remain generally stable throughout the study [14].

\section{Dosage and Administration}

Latanoprostene bunod $0.024 \%$ is approved in the USA for the reduction of IOP in patients with OAG or ocular hypertension [11]. The recommended dosage is one drop instilled in the conjunctival sac of the affected eye(s) once daily in the evening; this dosage should not be exceeded (as the instillation of PGAs more frequently than once daily has been shown to lessen the IOP-lowering effect). As latanoprostene bunod $0.024 \%$ contains the preservative benzalkonium chloride $0.2 \mathrm{mg} / \mathrm{mL}$, contact lenses should be removed prior to its topical administration and not reinserted until $15 \mathrm{~min}$ following installation. Latanoprostene bunod $0.024 \%$ may be instilled alongside other topical ophthalmic agents to lower IOP, although the medications should be administered $\geq 5$ min apart [11].

The use of latanoprostene bunod $0.024 \%$ is not recommended in patients aged $\leq 16$ years (because of potential safety concerns related to increased pigmentation following long-term use) [11]. In general, latanoprostene bunod $0.024 \%$ should not be used in patients with active intraocular inflammation (as it may exacerbate this condition) [11]. Local prescribing information should be consulted for detailed information regarding storage (with unopened bottles requiring refrigeration), use in special patient populations, and other warnings and precautions, including changes to pigmented tissues and eyelashes.

\section{Current Status of Latanoprostene Bunod $\mathbf{0 . 0 2 4 \%}$}

Several pharmacological options (the most frequently used initial intervention) are available for lowering IOP, with adverse events, cost, the dosing schedule and the degree of IOP lowering required influencing the treatment choice [19]. PGAs are considered first-line therapy in patients with primary $\mathrm{OAG}$, with latanoprost the most prescribed PGA in the USA [1]. Other agents include $\alpha$-adrenergic receptor agonists (e.g. brimonidine), $\beta$-blockers (e.g. timolol) and carbonic anhydrase inhibitors (e.g. brinzolamide) [19].

The NO-donating prostaglandin $\mathrm{F}_{2 \alpha}$ analogue latanoprostene bunod $0.024 \%$ is a novel IOP-lowering agent recently approved in the USA for the reduction of IOP in patients with OAG or ocular hypertension [11]. Following ocular instillation, latanoprostene bunod is rapidly metabolised to latanoprost acid and butanediol mononitrate (a NOdonating moiety) [Sect. 2]. It is thought to lower IOP via a dual mechanism of action: it increases aqueous humour outflow through the uveoscleral pathway (mediated by latanoprost acid) and increases the facility of aqueous humour outflow through the trabecular meshwork pathway (mediated by NO). The systemic concentrations of latanoprostene bunod and its metabolites is negligible (Sect. 2).

The IOP-lowering efficacy of the approved $0.024 \%$ concentration of latanoprostene bunod was noninferior to that of timolol $0.5 \%$ over 3 months in the multinational, phase III APOLLO and LUNAR studies and in a pooled analysis of these studies, with the superiority of latanoprostene bunod $0.024 \%$ over timolol $0.5 \%$ subsequently demonstrated in APOLLO and the pooled analysis (Sect. 3.1). Moreover, at all but the earliest timepoint evaluated in LUNAR, mean IOP was significantly lower with latanoprostene bunod $0.024 \%$ than timolol $0.5 \%$. The benefits of latanoprostene bunod $0.024 \%$ therapy seen during the active-controlled period were maintained during the openlabel safety extension periods of APOLLO (9 months) and LUNAR (3 months), with no apparent loss of IOP-lowering effect over time (Sect. 3.1). Results from APOLLO and LUNAR were confirmed in the phase III JUPITER study in Japanese patients (Sect. 3.2). IOP reductions were observed early (week 4) and maintained over the longer term (12 months) [Sect. 3.2]. It is worth noting that Japanese patients are known to have lower IOPs than non-Asian patients; indeed, the study and fellow treated eyes in JUPITER had a mean baseline IOP of 19.6 and $18.7 \mathrm{mmHg}$ [14], whereas the eyes in APOLLO and LUNAR had a mean baseline diurnal IOP of $26.4-26.7 \mathrm{mmHg}[12,13]$. Post hoc subgroup analyses (available as an abstract) [20] of data from APOLLO, LUNAR and JUPITER suggested that latanoprostene bunod $0.024 \%$ was effective in lowering IOP in patients with a baseline IOP within the normal range (i.e. $\leq 21 \mathrm{mmHg}$ in APOLLO and LUNAR and $\leq 19 \mathrm{mmHg}$ in JUPITER). Studies assessing the efficacy of latanoprostene bunod $0.024 \%$ specifically in patients with normotensive glaucoma, compared with other PGAs and over the long term would be of interest.

Latanoprostene bunod $0.024 \%$ was well tolerated over up to 12 months in adults with OAG or ocular hypertension, with the overall incidence of ocular adverse events 
generally comparable with those of other PGAs (Sect. 4). Most ocular TEAEs were mild or moderate in severity, with conjunctival hyperaemia being the most frequently reported ocular TEAE. The incidence of adverse events of special interest (eyelid and iris pigmentation, eyelash growth) was low (Sect. 4).

In conclusion, current evidence indicates once-daily latanoprostene bunod $0.024 \%$ is an effective and well tolerated treatment option for the reduction of IOP in adults with OAG or ocular hypertension.

\section{Data Selection Latanoprostene bunod: 76 records identified \\ Duplicates removed \\ Excluded during initial screening (e.g. press releases; news reports; not relevant drug/indication; preclinical study; reviews; case reports; not randomized trial) \\ Excluded during writing (e.g. reviews; duplicate data; small patient number; nonrandomized/phase I/II trials) \\ Cited efficacy/tolerability articles \\ Cited articles not efficacy/tolerability \\ Search Strategy: EMBASE, MEDLINE and PubMed from 1946 to present. Clinical trial registries/databases and websites were also searched for relevant data. Key words were Latanoprostene bunod, BOL-303259-X, Vyzulta, Vesneo, NCX-116. Records were limited to those in English language. Searches last updated 30 April 2018}

Acknowledgements During the peer review process, the manufacturer of latanoprostene bunod $0.024 \%$ was also offered an opportunity to review this article. Changes resulting from comments received were made on the basis of scientific and editorial merit.

\section{Compliance with Ethical Standards}

Funding The preparation of this review was not supported by any external funding.

Conflict of interest Sheridan Hoy is a salaried employee of Adis/ Springer, is responsible for the article content and declares no relevant conflicts of interest.

Open Access This article is distributed under the terms of the Creative Commons Attribution-NonCommercial 4.0 International License (http://creativecommons.org/licenses/by-nc/4.0/), which permits any noncommercial use, duplication, adaptation, distribution and reproduction in any medium or format, as long as you give appropriate credit to the original author(s) and the source, provide a link to the Creative Commons license and indicate if changes were made.

\section{References}

1. Liebmann JM, Lee JK. Current therapeutic options and treatments in development for the management of primary open-angle glaucoma. Am J Manag Care. 2017;23(15 Suppl):S279-92.
2. Abu-Hassan DW, Acott TS, Kelley MJ. The trabecular meshwork: a basic review of form and function. J Ocul Biol. 2014;2(1). http:// fulltextarticles.avensonline.org/JOCB-2334-838-02-0017.html.

3. Aptel F, Chiquet C, Romanet J-P. Intraocular pressure-lowering combination therapies with prostaglandin analogues. Drugs. 2012;72(10):1355-71.

4. Holló G, Katsanos A, Boboridis KG, et al. Preservative-free prostaglandin analogs and prostaglandin/timolol fixed combinations in the treatment of glaucoma: efficacy, safety and potential advantages. Drugs. 2018;78(1):39-64.

5. Lu LJ, Tsai JC, Liu J. Novel pharmacologic candidates for treatment of primary open-angle glaucoma. Yale J Biol Med. 2017;90(1):111-8.

6. Cavet ME, Vittitow JL, Impagnatiello F, et al. Nitric oxide (NO): an emerging target for the treatment of glaucoma. Invest Ophthalmol Vis Sci. 2014;55(8):5005-15.

7. Cavet ME, DeCory HH. The role of nitric oxide in the intraocular pressure lowering efficacy of latanoprostene bunod: review of nonclinical studies. J Ocul Pharmacol Ther. 2018;34(1-2):52-60.

8. Cavet ME, Vollmer TR, Harrington KL, et al. Regulation of endothelin-1-induced trabecular meshwork cell contractility by latanoprostene bunod. Invest Ophthalmol Vis Sci. 2015;56(6):4108-16.

9. Saeki T, Tsuruga H, Aihara M, et al. Dose-response profile of PF03187207 (PF-207) and peak IOP lowering response following single topical administration to FP receptor knockout mice vs. wild type mice [abstract no. 4064]. Invest Ophthalmol Vis Sci. 2009;50(13).

10. Krauss $\mathrm{AH}$, Impagnatiello $\mathrm{F}$, Toris $\mathrm{CB}$, et al. Ocular hypotensive activity of BOL-303259-X, a nitric oxide donating prostaglandin F2 $\alpha$ agonist, in preclinical models. Exp Eye Res. 2011;93(3):250-5.

11. Bausch \& Lomb Inc. VYZULTA (latanoprostene bunod ophthalmic solution) $0.024 \%$, for topical ophthalmic use: US prescribing information. 2017. http://www.fda.gov/. Accessed 30 Jan 2018.

12. Weinreb RN, Scassellati Sforzolini B, Vittitow J, et al. Latanoprostene bunod $0.024 \%$ versus timolol maleate $0.5 \%$ in subjects with open-angle glaucoma or ocular hypertension: the APOLLO study. Ophthalmology. 2016;123(5):965-73.

13. Medeiros FA, Martin KR, Peace J, et al. Comparison of latanoprostene bunod $0.024 \%$ and timolol maleate $0.5 \%$ in open-angle glaucoma or ocular hypertension: the LUNAR study. Am J Ophthalmol. 2016;168:250-9.

14. Kawase K, Vittitow JL, Weinreb RN, et al. Long-term safety and efficacy of latanoprostene bunod $0.024 \%$ in Japanese subjects with open-angle glaucoma or ocular hypertension: the JUPITER study. Adv Ther. 2016;33(9):1612-27.

15. Weinreb RN, Ong T, Scassellati Sforzolini B, et al. A randomised, controlled comparison of latanoprostene bunod and latanoprost $0.005 \%$ in the treatment of ocular hypertension and open angle glaucoma: the VOYAGER study. Br J Ophthalmol. 2015;99(6):738-45.

16. Liu JH, Slight JR, Vittitow JL, et al. Efficacy of latanoprostene bunod $0.024 \%$ compared with timolol $0.5 \%$ in lowering intraocular pressure over 24 hours. Am J Ophthalmol. 2016;169:249-57.

17. Weinreb RN, Liebmann JM, Martin KR, et al. Latanoprostene bunod $0.024 \%$ in subjects with open-angle glaucoma or ocular hypertension: pooled phase 3 study findings. J Glaucoma. 2018;27(1):7-15.

18. Kaufman PL. Latanoprostene bunod ophthalmic solution $0.024 \%$ for IOP lowering in glaucoma and ocular hypertension. Expert Opin Pharmacother. 2017;18(4):433-44.

19. Prum BE Jr, Rosenberg LF, Gedde SJ, et al. Primary open-angle glaucoma Preferred Practice Pattern ${ }^{\circledR}$ guidelines. Ophthalmology. 2016;123(1):P41-111.

20. Fingeret M, Powell M, Vittitow J. Efficacy of latanoprostene bunod ophthalmic solution $0.024 \%$ in eyes with normal intraocular pressures [abstract]. In: American Academy of Optometry 95th Annual Meeting. 2016. 